\title{
Connectivity Orchestration in Multi-Provider Elastic Optical Networks
}

\author{
(Invited Paper) \\ Alessio Giorgetti, Francesco Paolucci, Piero Castoldi \\ Scuola Superiore Sant'Anna \\ Pisa, Italy \\ Email: a.giorgetti@santannapisa.it
}

\begin{abstract}
Next-generation $5 \mathrm{G}$ concepts are driving infrastructure providers to enable novel services for network operators, such as Virtual Network Function (VNF) and Network Function Virtualization (NFV). Inside a single infrastructure provider, several solutions are available to satisfy the $5 \mathrm{G}$ requirements using Software Defined Networking (SDN).

However, emerging industry verticals require geographicallydistributed services spanning multiple infrastructure providers. In this multi-provider context, additional complexity emerges to guarantee inter-operability. The orchestrator of each multidomain infrastructure (i.e., MDO), is emerging as the key element to offer services to a federation of infrastructure providers. In this scenario, the procedures for establishing inter-provider services are still an open research topic.

This work proposes and evaluates two different procedures to enable multi-provider connectivity service exploiting distributed communication among MDOs. The two procedures are evaluated by means of simulations in a federated multi-provider network based on the Elastic Optical Network technology.
\end{abstract}

\section{INTRODUCTION}

The stringent requirements driven by next-generation $5 \mathrm{G}$ concepts are driving infrastructure providers to enable novel services for network operators, such as network slicing functions and Virtual Network Function (VNF) services [1]. To this end, Software Defined Networking (SDN) and Network Function Virtualization (NFV) are evolving in recent years, decoupling control and data plane and moving network functions from hardware-specific devices to software-based genericpurpose equipments, e.g., in data center networks.

Inside a single infrastructure provider, several solutions enabling 5G requirements (i.e., low latency, content-based caching, network slicing) will be available in the context of SDN-NFV framework. The development of Application Programming Interface (API)-based controllers and orchestrators able to dynamically coordinate the creation and the handling of virtual services, computing and storage resources, and communication networks for multi-tenant utilization is becoming attractive. Specifically, API-based controllers and orchestrators are in advanced phase of development, that can effectively coordinate creation and concatenation of virtual services (including computing, storage and network resources).

However, emerging vertical industry approaches (e.g., automotive-based logistics, energy grids, e-health services) may require geographically-distributed VNF slicing shared with more than a single infrastructure provider [2]. In this multi-provider context, additional complexity is required to handle shared and inter-operable control and management sessions through different operators implementing their own administrative and business strategies, along with the need to preserve high degrees of confidentiality and security [2]. Therefore, the orchestrator of each multi-domain network infrastructure, i.e., the Multi-domain Orchestrator (MDO), is emerging as the key element able to offer and coordinate service instances, to a federation of infrastructure providers while monitoring and guaranteeing agreed Service Level Agreements (SLAs) [3]. In such a context, connectivity functions and procedures among MDOs requires careful evaluation and additional constraints with respect to the control of a singleprovider infrastructure.

In this work, we present and evaluate two different procedures to enable multi-provider connectivity provisioning resorting to the stateful Hierarchical Path Computation Element (PCE) architecture [4]. The peer and the source-hierarchical procedures are proposed and described. The two procedures are evaluated extensively by means of simulations in a federated multi-provider network scenario based on the IP/MPLS over Elastic Optical Network technology.

\section{RELATED WORK}

Several TE solutions have been proposed in the past years to be applied in GMPLS-based single-domain WSONs. In such networks the routing protocol (e.g., OSPF-TE) advertises connectivity and resource availability information [5], [6]. Therefore effective path computation can be performed either locally by the source node or by a dedicated PCE. On the other hand, the first solutions proposed for the multi-domain scenario were based on Border Gateway Protocol (BGP) not considering resource availability. To address this issue, the PCE architecture has been extended to support inter-domain path computation using a coordinated PCEP communication process among PCEs. As an example the Backward Recursive Path Computation (BRPC) procedure has been proposed to identify the optimal path considering also current resource utilization [7]. However, those procedures assume that the sequence of domains to be traversed is known in advance. 

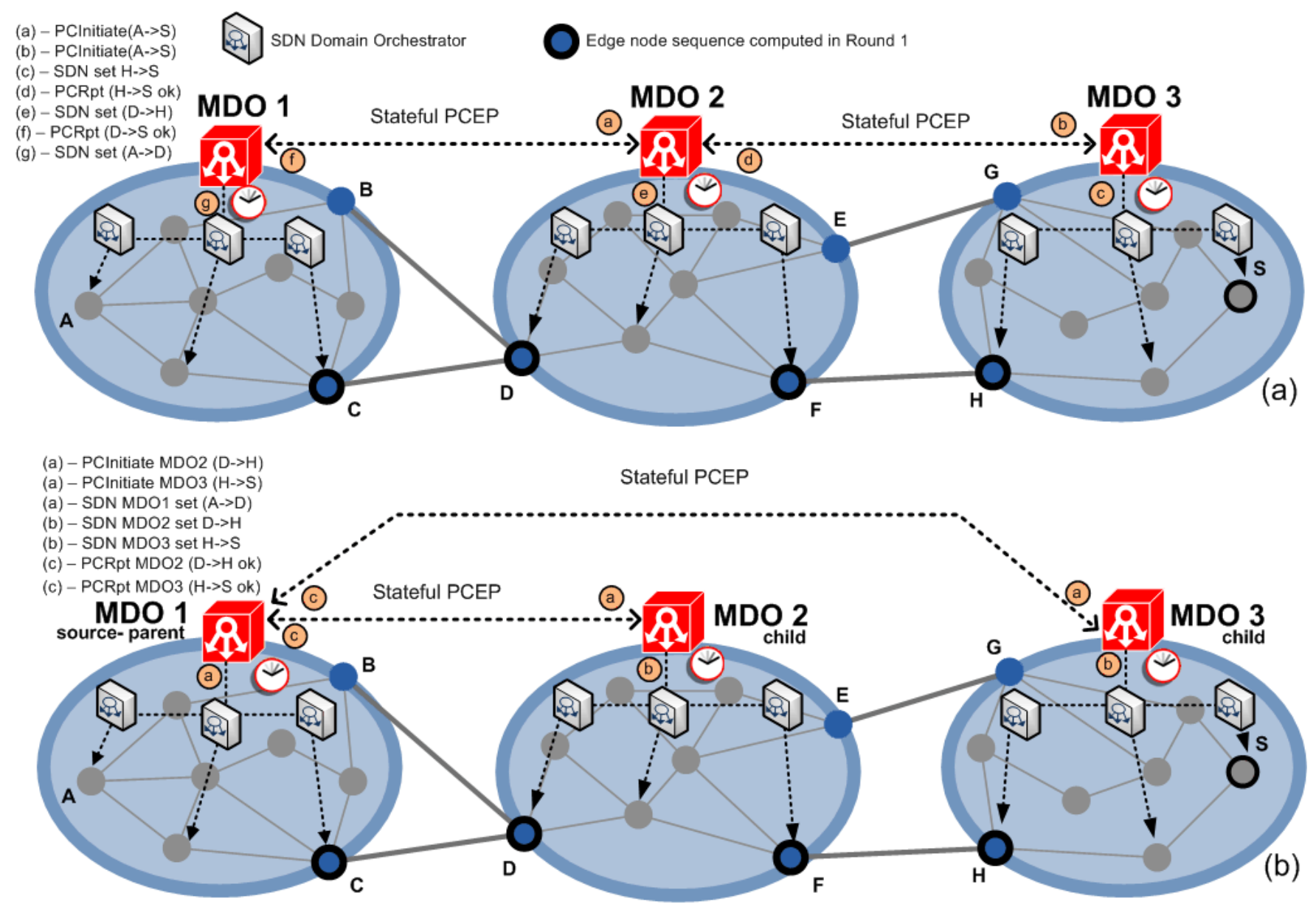

Fig. 1. Proposed architecture: peer (a) and source-hierarchical (b) procedures, for establishing inter-provider connectivity.

More recently, the H-PCE architecture has been proposed to coordinate the inter-domain path computation including the selection of the sequence of domains [8], [9]. Originally, the Parent PCE (pPCE) has been designed with a Hierarchical TED (H-TED) including only inter-domain connectivity information (e.g., resource availability on inter-domain links), to determine the sequence of domains. However, several works demonstrated that intra-domain resource information is required to achieve effective inter-domain path computation [4], [10], [11]. Therefore, several solutions have been proposed where the pPCE is allowed to dynamically ask Child PCEs (cPCEs) for the path computation of the several edge-to-edge segments considering intra-domain resource information. However, this solution requires high number of messages between pPCE and cPCEs. The works in [11]-[13] propose a reduction of the generated PCEP messages but imply a degradation of the lightpath blocking probability. However, some experimental works demonstrated that the aforementioned procedures are feasible in realistic multi-domain WSONs even if they significantly increase the PCEP traffic [14], [15].

More recently new proposals emerged to enable effective inter-domain path computation without requiring extensive communication among PCEs. Specifically, the inclusion of intra-domain information directly in the H-TED has been proposed [16], where such information is provided to the pPCE by resorting to the recently proposed TE Link State Information extension to BGP (BGP-LS), as experimentally demonstrated in [17], [18]. However, this BGP-LS solution is still based on OSPF-TE advertisements and can therefore suffer in dynamic traffic scenarios such as during restoration. As an example, the work in [19] dynamic restoration in multi-domain EONs and demonstrates a blocking probability of several percentage points also with very low traffic load. Therefore, novel solutions have been proposed to update the $\mathrm{H}$-TED maintained at the pPCE making a limited use of BGP-LS updates. That solutions further reduces the amount of messages required among PCEs while making the BGPLS updates independent of OSPF-TE and also decreases the blocking probability during provisioning and restoration [20].

However, that kind of solutions based on the H-PCE architecture cannot be easily extended to a multi-provider scenario mainly because of security and confidentiality issues that can emerge when exchange of detailed network resource utilization are required. Within the multi-provider scenario a business-driven PCE is proposed in [21]. The approach is based on the introduction of business metrics, such as SLA 


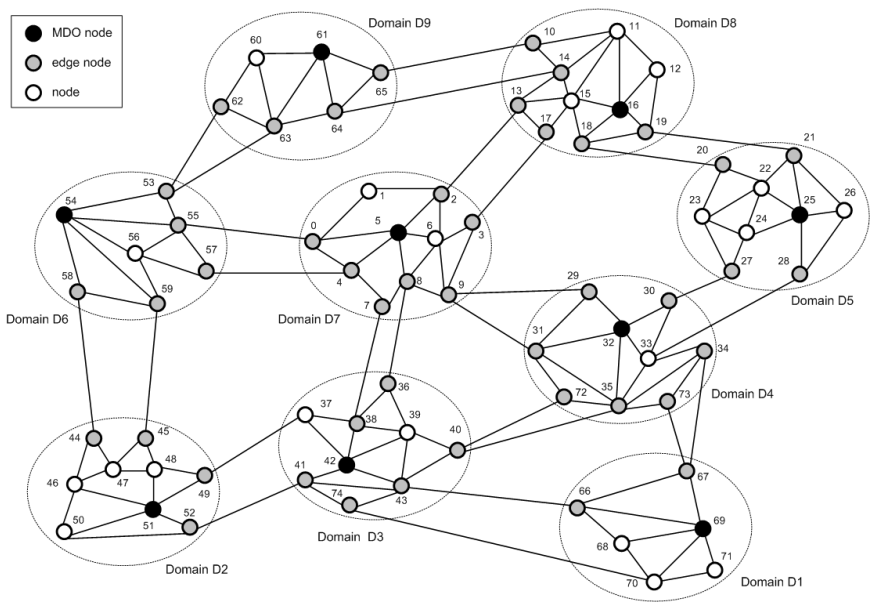

Fig. 2. Multi-provider EON test network topology.

matching and pricing, in order to select either the domain sequence and the BRPC end-to-end path. In [22], a backwardcompatible mechanism is proposed to enable both domain summarization and multi-path routing, by encapsulating link disjoint paths onto a single virtual path and by providing multiple virtual intra-domain topologies. The work in [3] proposes an orchestrator architecture to handle virtual services across different operators exploiting a multi-domain PCE as orchestrator module for each provider. Finally, a number of studies discussed smart mechanisms to allow authenticated peer PCE to infer critical intra-domain information of adjacent domains through licit PCEP requests exploiting concepts such as signature-based and anomaly-based detection and trust management mechanisms [23], [24].

The two procedures proposed in this paper enable multiprovider connectivity provisioning resorting to the stateful Hierarchical Path Computation Element (PCE) architecture. Specifically, in the peer procedure each PCEs only communicate with its neighbours, while in the source-hierarchical procedures the PCE of the source provider network temporarily assumes the role of pPCE only for the specific connection request.

\section{CONNECTIVITY ESTABLISHMENT PROCEDURES}

The considered federated multi-provider network architecture is illustrated in Fig. 1. A number of infrastructure providers are supposed to join the federation. Each provider have full administrative control on its own infrastructure. Each infrastructure is supposed to be heterogeneous and composed of several domains (e.g., 5G front-haul access, metro, core) deploying different control technologies (e.g., (G)MPLS, SDN). Each domain may also include IT resources, e.g., datacenters and is controlled by a local domain orchestrator. In order to enable multi-provider federation, a single MDO per provider is supposed to act at the top hierarchy of the domain orchestrators owned by the same provider and is in charge of establishing peering sessions with federated MDOs and coordinate resource provisioning when inter-provider services are required.
Each MDO provides the list of available services through market-place application to the other federated MDOs. Focusing on connectivity services, each MDO stores the intradomain topology and establishes a BGP peering session with the federated MDOs to assure best-effort reachability. Moreover, MDO runs BGP-LS instances exchanging abstracted intra-domain topology and SLAs [25]. In order to achieve inter-provider TE solutions while preserving confidentiality, the abstracted intra-domain topology provides a simplified representation of the physical topology, e.g., including only edge nodes interconnected by a set of virtual intra-domain links provided with aggregated TE information [20].

Our proposal for multi-provider architecture extends the state of the art procedures adopted in multi-domain networks, where path computation and instantiation is delegated to a stateful PCE. Specifically, the two proposed inter-provider establishment procedures rely on peer and hierarchical relationships among MDOs, respectively. Both proposed procedures are performed in two rounds. In the first round, the end-to-end path computation is performed; in the second round end-toend service is established. The peer MDO procedure is based on the Backward Recursive PCE-based computation (BRPC), while the source-hierarchical MDO procedure is based on the Hierarchical PCE architecture, in which a single parent PCE coordinates the path computation among multiple child PCEs [4]. However, in multi-provider scenario, the delegation of connectivity provisioning to a single parent MDO is unrealistic. Thus, in the source-hierarchical procedure we propose that the role of parent MDO is played alternatively by the MDO of the source network.

In the peer procedure, a PCEP session is established only between contiguous MDOs (e.g., MDO1 and MDO2 in Fig. 1(a)). Provider sequence selection is performed by resorting to BGP-LS information exchanged among MDOs. Then, stateful BRPC is applied for the path computation resorting to PCEP communication between contiguous MDOs. When the detailed path has been computed using BRPC, connectivity is instantiated along the computed path using signaling protocols specific of each network domain (e.g., (G)MPLS, SDN protocols). The instantiation procedure is illustrated in Fig. 1(a) for a connection A-S traversing providers 1, 2, 3. Following the computed path, PCInitiate messages are forwarded up to the destination MDO (step a and step b). Destination MDO (i.e., MDO3) performs instantiation by triggering all involved SDN domain orchestrators (step c). After successful establishment of local segment $\mathrm{MDO} 3$ provides a PCReport message to MDO2 (step d). Upstream MDOs perform the same procedure (step e and step f) until the source MDO is reached, thus triggering source domain setup (step g).

In the source-hierarchical procedure each MDO maintains a PCEP session towards each federated MDOs (e.g, MDO1 towards MDO2 and MDO3 in Fig. 1(b)). When a new interprovider connection request arrives to the source MDO, this MDO acts as parent MDO towards the federated MDOs involved in the provider sequence. Path computation is performed using aggregated TE information provided by BGP- 


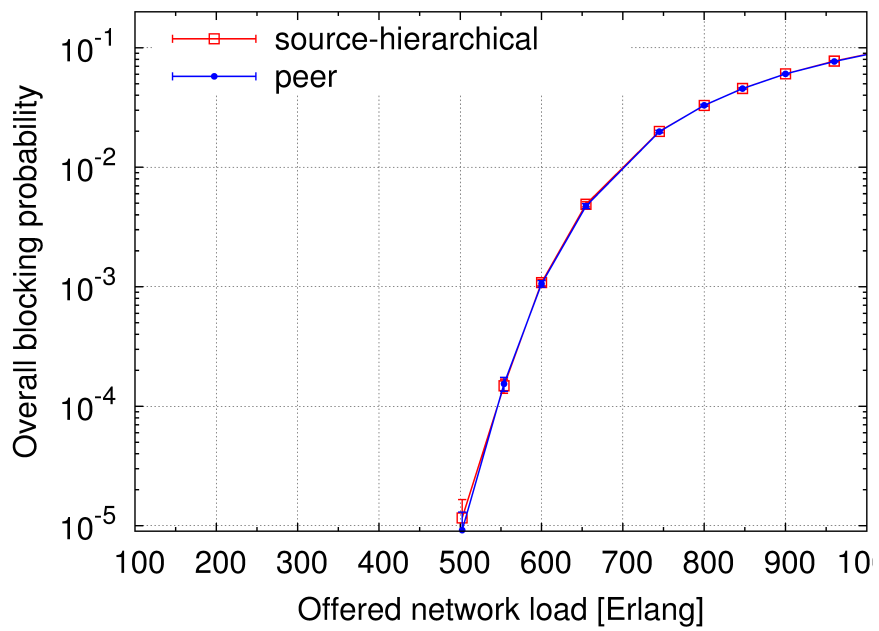

Fig. 3. Simulation results: connection blocking probability vs. offered network load.

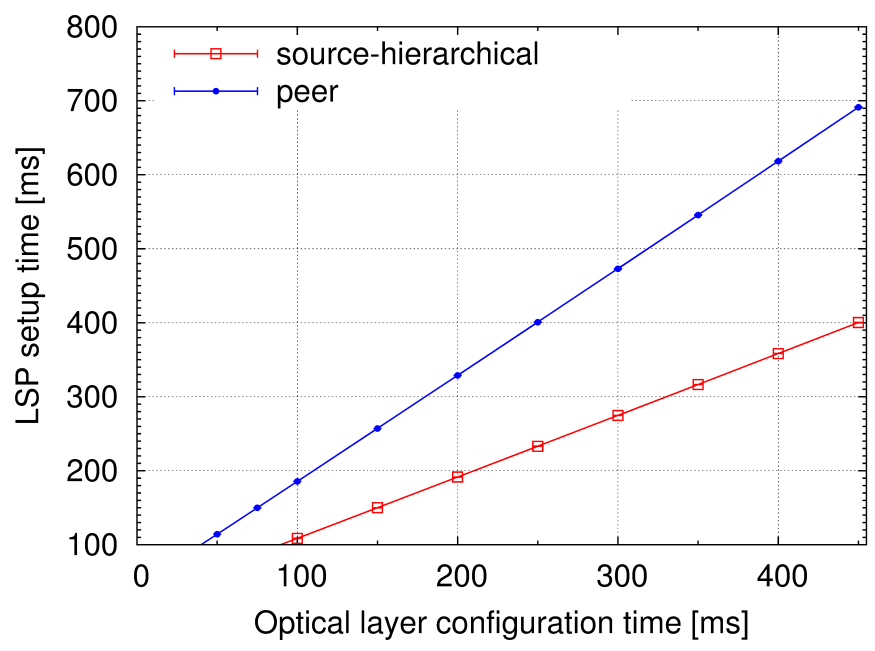

Fig. 4. Simulation results: connection establishment time vs. time required to configure an EON node.

LS and following the standard HPCE procedure (i.e., source MDO can ask specific resource utilization to optimize the path computation). Then, path instantiation is performed using a parallelized procedure, as shown in Fig. 1(b). Specifically, the source MDO (i.e., MDO1) contacts all the involved transit and egress MDOs by sending PCInitiate messages with the ERO segment of the local path segment (step a). Moreover, the source MDO starts provisioning in its domain (step a). Upon reception of the PCInitiate message, each MDO performs the provisioning in the local infrastructure (step b). At the end of each segment provisioning, PCReport messages are provided by each MDOs to the source MDO (step c). Note that, in this procedure, parent-child relationship is not static, but change dynamically for each connection request. This approach is particularly compatible with advanced trust management mechanisms [24].

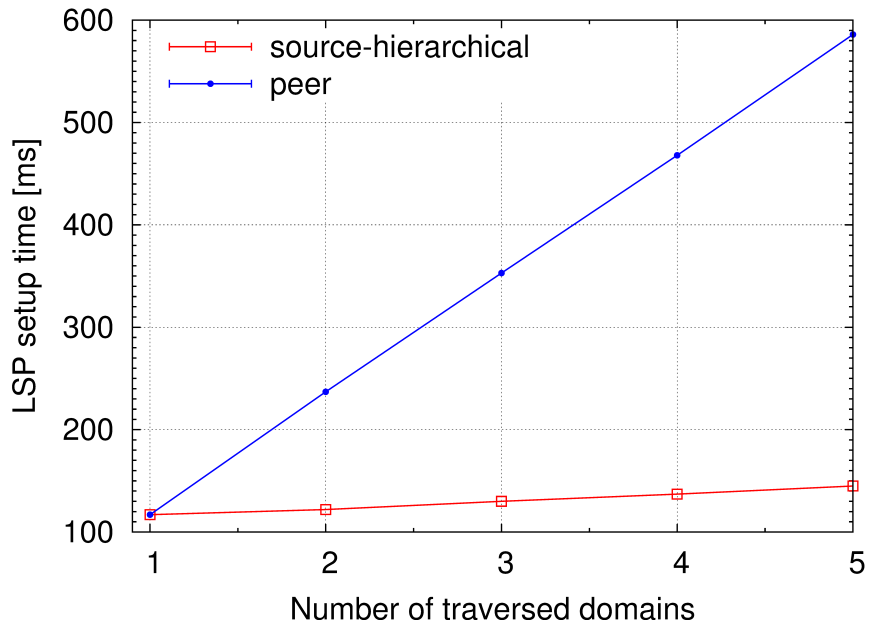

Fig. 5. Simulation results: connection establishment time vs. number of traversed providers.

\section{Simulation REsults}

The described procedures are evaluated using a custom built event-driven $\mathrm{C}++$ simulator. The considered multi-provider MPLS-over-EON topology, illustrated in Fig. III, includes 9 providers, 75 nodes and 145 bidirectional links with 256 frequency slots per direction [20]. Each connection requires the utilization of 3 or 9 frequency slices. It is supposed that OEO conversion is applied at edge nodes so that spectrum contiguity and continuity constraints are only enforced within each provider network. Each MDO is co-located within a node. The traffic is uniformly distributed among node pairs and connectivity requests arrive following a Poisson process, the mean holding time is fixed to 12 hours. For both procedures, routing selects the least congested path among the paths crossing the minimum number of domains. Spectrum assignment is first-fit.

Fig. III illustrates the connection blocking probability as a function of the offered network load, and demonstrates that, utilizing the same path computation, the two instantiation procedures achieve the same blocking probability. Fig. III illustrates the connection setup time as a function of time required to configure an EON node. This result show that both procedures require an increasing setup time when the time required to configure the optical layer increases (typical configuration time may vary from few tens of milliseconds, i.e., MEMS based optical nodes, to hundreds of milliseconds, i.e., LCOS based optical nodes) [26]. However, setup time provided by the source-hierarchical procedure is always lower and increasing with a lower slope. Fig. III illustrates the connection setup time as a function of the number of providers traversed by the specific connection. This result show that, thanks to the parallelization of the establishment the sourcehierarchical procedure provides a setup time that is almost independent on the number of traversed domains. Conversely, from this point of view, peer scheme is not scalable due to the adopted sequential establishment procedure. 


\section{CONCLUSIONS}

Two instantiation procedures are proposed to be used in federated multi-provider networks. Simulation results obtained in IP/MPLS over EON network scenario, demonstrated that the proposed source-hierarchical procedure, introducing the idea of alternating source-parent MDO, is able to guarantee short establishment time especially when many provider networks have to be traversed.

\section{ACKNOWLEDGMENT}

This work has been partially supported by the EU H2020 project 5G Exchange (Grant Agreement no. 671636).

\section{REFERENCES}

[1] K. Samdanis et al., "From network sharing to multi-tenancy: The 5G network slice broker," IEEE Commun. Mag., vol. 54, no. 7, pp. 32-39, 2016.

[2] L. Contreras et al., "Orchestration of crosshaul slices from federated administrative domains," in Proc. EuCNC, Athens, 2016.

[3] A. Sgambelluri et al., "Orchestration of network services across multiple operators: The 5g exchange prototype," in 2017 European Conference on Networks and Communications (EuCNC), Jun. 2017, pp. 1-5.

[4] F. Paolucci, F. Cugini, A. Giorgetti, N. Sambo, and P. Castoldi, "A survey on the path computation element (PCE) architecture," IEEE Commun. Surveys Tuts., vol. 15, no. 4, pp. 1819-1841, 2013.

[5] M. Tornatore, F. De Grandi, R. Muoz, R. Martinez, R. Casellas, and A. Pattavina, "Effects of outdated control information in control-planeenabled optical networks with path protection," J. Opt. Commun. Netw., vol. 1, no. 2, pp. A194-A204, Jul. 2009.

[6] A. Giorgetti, F. Cugini, N. Sambo, F. Paolucci, N. Andriolli, and P. Castoldi, "Path state-based update of PCE traffic engineering database in wavelength switched optical networks," IEEE Commun. Lett., vol. 14, no. 6, pp. 575-577, Jun. 2010.

[7] J. Vasseur, "A backward-recursive PCE-based computation (BRPC) procedure to compute shortest constrained inter-domain traffic engineering label switched paths," IETF RFC 5441, Apr. 2009.

[8] D. King and A. Farrel, "The application of the path computation element architecture to the determination of a sequence of domains in MPLS and GMPLS," IETF RFC 6805, Nov. 2012.

[9] D. Siracusa, S. Grita, G. Maier, A. Pattavina, F. Paolucci, F. Cugini, and P. Castoldi, "Domain sequence protocol (dsp) for pce-based multidomain traffic engineering," J. Opt. Commun. Netw., vol. 4, no. 11, pp. 876-884, 2012.

[10] S. Spadaro, J. Perello, G. Hernandez-Sola, A. Moreno, F. Agraz, J. Comellas, and G. Junyent, "Analysis of traffic engineering information dissemination strategies in PCE-based multi-domain optical networks," in Proc. ICTON, Jun. 2010.

[11] A. Giorgetti, F. Paolucci, F. Cugini, and P. Castoldi, "Impact of intradomain information in GMPLS-based WSONs with hierarchical PCE," in Tech. Dig. OFC/NFOEC, Mar. 2012.
[12] G. Hernandez-Sola, J. Perello, F. Agraz, S. Spadaro, J. Comellas, and G. Junyent, "Scalable hybrid path computation procedure for PCE-based multi-domain WSON networks," in Proc. ICTON, Jun. 2011.

[13] S. Shang, X. Zheng, H. Zhang, N. Hua, and H. Zhang, "A hierarchical path computation element (PCE)-based routing algorithm in multidomain WDM networks," in Proc. ACP, Dec. 2010.

[14] R. Casellas, R. Munoz, and R. Martinez, "Lab trial of multi-domain path computation in GMPLS controlled WSON using a hierarchical PCE," in Tech. Dig. OFC/NFOEC, Mar. 2011.

[15] J. Zhang, Y. Zhao, X. Chen, Y. Ji, M. Zhang, H. Wang, Y. Zhao, Y. Tu, Z. Wang, and H. Li, "The first experimental demonstration of a dream-based large-scale optical transport network with 1000 control plane nodes," Optics express, vol. 19, no. 26, pp. B746-B755, 2011.

[16] H. Gredler, J. Medved, S. Previdi, A. Farrel, and S. Ray, "North-Bound Distribution of Link-State and Traffic Engineering (TE) Information Using BGP,' RFC 7752 (Proposed Standard), pp. 1-48, Mar. 2016.

[17] M. Cuaresma, F. Munoz del Nuevo, S. Martinez, A. Mayoral, O. Gonzalez de Dios, V. Lopez, and J. Fernandez-Palacios, "Experimental demonstration of H-PCE with BPG-LS in elastic optical networks," in Proc. ECOC, Sep. 2013.

[18] O. Gonzalez de Dios, R. Casellas, R. Morro, F. Paolucci, V. Lopez, R. Martinez, M. R., V. R., and P. Castoldi, "First multi-partner demonstration of BGP-LS enabled inter-domain EON control with H-PCE," in Tech. Dig. OFC, Mar. 2015.

[19] X. Li, S. Huang, Y. Zhao, M. Zhang, D. Wang, Z. Wang, J. Zhang, and W. Gu, "Combination of distributed and centralized control architecture based on PCE protocol for large scale multi-domain elastic optical networks," in Proc. ICOCN, Nov. 2014.

[20] A. Giorgetti, "Proactive H-PCE architecture with BGP-LS update for multidomain elastic optical networks [invited]," J. Opt. Commun. Netw. vol. 7, no. 11, pp. 1-9, 2015.

[21] N. B. Djarallah, H. Pouyllau, N. L. Sauze, and R. Douville, "Businessdriven pce for inter-carrier qos connectivity services," in 2011 Future Network Mobile Summit, Jun. 2011.

[22] X. Chen, M. Chamania, and A. Jukan, "A backward-compatible interdomain multipath routing framework," in 2011 IEEE Conference on Computer Communications Workshops (INFOCOM WKSHPS), Apr. 2011.

[23] F. Paolucci, M. Gharbaoui, A. Giorgetti, F. Cugini, B. Martini, L. Valcarenghi, and P. Castoldi, "Preserving confidentiality in pce-based multidomain networks," IEEE/OSA Journal of Optical Communications and Networking, vol. 3, no. 5, pp. 465-474, May 2011.

[24] M. Gharbaoui, B. Martini, C. Fung, F. Paolucci, A. Giorgetti, and P. Castoldi, "An incentive-compatible and trust-aware multi-provider path computation element (pce)," Computer Networks, vol. 108, pp. 40 $-54,2016$.

[25] F. Paolucci et al., "Interoperable multi-domain delay-aware provisioning using segment routing monitoring and bgp-ls advertisement," in Proc. ECOC, Dusseldorf, 2016.

[26] A. Giorgetti et al., "Dynamic restoration with GMPLS and SDN control plane in elastic optical networks [invited]," J. Opt. Commun. Netw., vol. 7, no. 2, pp. A174-A182, 2015. 